\title{
Apparent Reversal of Molecular Orbitals Reveals Entanglement
}

\author{
Ping Yu \\ Institute of Experimental and Applied Physics, University of Regensburg, 93053 Regensburg, Germany \\ and School of Physical Science and Technology, ShanghaiTech University, 201210 Shanghai, China \\ Nemanja Kocić and Jascha Repp* \\ Institute of Experimental and Applied Physics, University of Regensburg, 93053 Regensburg, Germany
}

Benjamin Siegert and Andrea Donarini ${ }^{\dagger}$

Institute of Theoretical Physics, University of Regensburg, 93053 Regensburg, Germany

(Received 7 April 2017; revised manuscript received 10 June 2017; published 1 August 2017)

\begin{abstract}
The frontier orbital sequence of individual dicyanovinyl-substituted oligothiophene molecules is studied by means of scanning tunneling microscopy. On $\mathrm{NaCl} / \mathrm{Cu}(111)$, the molecules are neutral, and the two lowest unoccupied molecular states are observed in the expected order of increasing energy. On $\mathrm{NaCl} / \mathrm{Cu}(311)$, where the molecules are negatively charged, the sequence of two observed molecular orbitals is reversed, such that the one with one more nodal plane appears lower in energy. These experimental results, in open contradiction with a single-particle interpretation, are explained by a manybody theory predicting a strongly entangled doubly charged ground state.
\end{abstract}

DOI: 10.1103/PhysRevLett.119.056801

For the use of single molecules as devices, engineering and control of their intrinsic electronic properties is all important. In this context, quantum effects such as electronic interference have recently shifted into the focus [1-7]. Most intriguing in this respect are electron correlation effects [8-14], which are intrinsically strong in molecules due to their small size [15-19].

In general, Coulomb charging energies strongly depend on the localization of electrons and hence, on the spatial extent of the orbitals they occupy. Therefore, the orbital sequence of a given molecule can reverse upon electron attachment or removal if some of the frontier orbitals are strongly localized while others are not, like in, e.g., phthalocyanines [20-24]. Coulomb interaction may also lead to much more complex manifestations such as quantum entanglement of delocalized molecular orbitals.

Here, we show that the energy spacing of the frontier orbitals in a single molecular wire of individual dicyanovinyl-substituted quinquethiophene (DCV5T) can be engineered to achieve near degeneracy of the two lowest-lying unoccupied molecular orbitals, leading to a strongly entangled ground state of DCV5T ${ }^{2-}$. These orbitals are the lowest two of a set of particle-in-a-box-like states and differ only by one additional nodal plane across the center of the wire. Hence, according to the fundamental oscillation theorem of the Sturm-Liouville theory, their sequence has to be set with an increasing number of nodal planes, which is one of the basic principles of quantum mechanics $[25,26]$. This is evidenced and visualized from scanning tunneling microscopy (STM) and spectroscopy (STS) of DCV5T on ultrathin insulating films. Upon lowering the substrate's work function, the molecule becomes charged, leading to a reversal of the sequence of the two orbitals. The fundamental oscillation theorem seems strikingly violated since the state with one more nodal plane appears lower in energy. This contradiction can be solved, though, by considering intramolecular correlation leading to a strong entanglement in the ground state of DCV5T ${ }^{2-}$.

The experiments were carried out with a home-built combined STM and atomic force microscopy (AFM) using a qPlus sensor [27] operated in ultrahigh vacuum at a temperature of $6 \mathrm{~K}$. Bias voltages are applied to the sample. All AFM data, $d I / d V$ spectra, and maps were acquired in constant-height mode. Calculations of the orbitals and effective single-particle electronic structure were performed within the density functional theory (DFT) as implemented in the SIESTA code [28] and are based on the Perdew-BurkeErnzerhof generalized gradient approximation (GGA). The many-body eigenstates are determined from a diagonalization of the many-body model Hamiltonian $H_{\text {mol }}$, which is defined further below in the main text. Based on these, STM-image and spectra simulations were performed within a Liouville approach for the density matrix $\rho$. See Supplemental Material [29] (SM) for more details.

The molecular structure of DCV5T, shown in Fig. 1(a), consists of a quinquethiophene (5T) backbone and a dicyanovinyl (DCV) moiety at each end. The delocalized electronic system of polythiophene and oligothiophene enables conductance of this material $[30,49,50]$. The lowest unoccupied orbital of each of the thiophene rings couples electronically to its neighbors and forms a set of particle-ina-box-like states $[51,52]$. The lowest-energy unoccupied molecular orbital (LUMO) to LUMO + 1 level spacing of the quinquethiophene (5T) backbone is approximately 


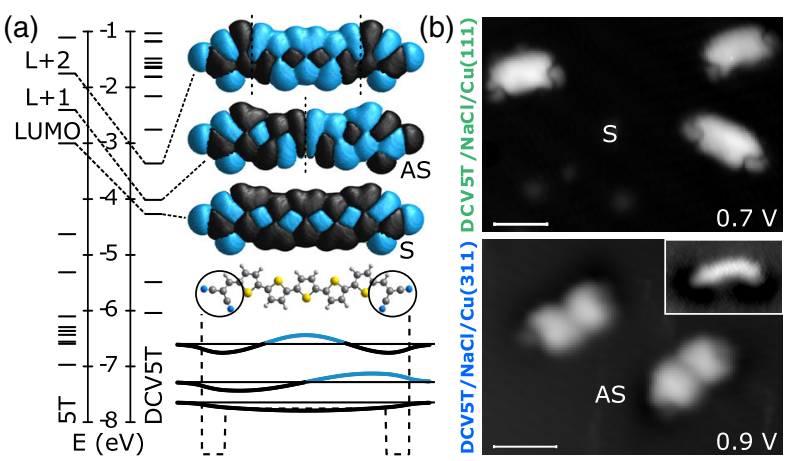

FIG. 1. (a) Molecular structure and density-functional theory based calculations of the electronic structure of 5T and DCV5T. The panel depicts the molecular structure, the calculated orbitals, and energies for the LUMO, LUMO + 1, and LUMO + 2 as indicated. The orbitals are depicted as contours of constant probability density. The LUMO and LUMO +1 orbitals derive from the thiophene subunit's LUMO. They are the lowest two of a set of particle-in-a-box-like states and differ only by one additional nodal plane. Whereas the LUMO to LUMO +1 energy difference is approximately $0.7 \mathrm{eV}$ for $5 \mathrm{~T}$, this difference is drastically reduced in the case of DCV5T. The basic principle of level engineering is illustrated for a one dimensional quantum box. (b) STM images of the first DCV5T electronic resonance for $\mathrm{NaCl} / \mathrm{Cu}(111)$ (top) and $\mathrm{NaCl} / \mathrm{Cu}(311)$ (bottom) as substrates. The inset shows a STM image at a voltage below the first molecular resonance scale bar $2 \mathrm{~nm}$.

$0.7 \mathrm{eV}$ [51], which is in good agreement with the energy difference calculated for free 5T based on DFT, as shown in Fig. 1(a), left. This DFT-based calculation also confirms the nature of the LUMO and LUMO +1 orbitals, both deriving from the single thiophene's LUMOs and essentially differing only by one additional nodal plane across the center of the molecule. To enable the emergence of correlation and thus, level reordering, we have to bring these two states closer to each other. This is achieved by substituting dicyanovinyl moieties with larger electron affinity at each end of the molecular wire. As the orbital density of the higher-lying particle-in-a-box-like state, namely $\mathrm{LUMO}+1$, has more weight at the ends of the molecule, it is more affected by this substitution than the lowest state, the LUMO. This is evidenced by corresponding calculations of DCV5T, for which, the LUMO to LUMO + 1 energy difference is reduced by more than a factor of two, see Fig. 1(a), left. The increased size of DCV5T may also contribute to the reduced level spacing. For the rest of this Letter, we concentrate on the LUMO and LUMO + 1 orbitals only. To avoid confusion, we refrain from labeling the orbitals according to their sequence but instead according to their symmetry with respect to the mirror plane perpendicular to the molecular axis as symmetric (S) and antisymmetric (AS). Hence, the former LUMO and the LUMO +1 are the S and AS states, respectively.

To study the energetic alignment of the orbitals as well as their distribution in real space, we employ ultrathin $\mathrm{NaCl}$ insulating films to electronically decouple the molecules from the conductive substrate [31]. It has been previously shown that in these systems, the work function can be changed by using different surface orientations of the underlying metal support $[31,53,54]$. Importantly, this does not affect the (100)terminated surface orientation of the $\mathrm{NaCl}$ film, such that the local chemical environment of the molecule remains the same, except for the change of the work function.

However, in the present case, this alone has a dramatic effect on the electronic structure of the molecular wires, as is evidenced in Fig. 1(b). There, the STM images are shown for voltages corresponding to the respective lowest-lying molecular resonances at positive sample voltage for DCV5T adsorbed on $\mathrm{NaCl} / \mathrm{Cu}(111)$ (top panel) and $\mathrm{NaCl} / \mathrm{Cu}(311)$ (bottom panel). They both show a hotdog-like appearance of the orbital density, in which a central lobe is hidden inside outer lobes, as was discussed previously [51,55]. However, whereas the orbital density of DCV5T/ $\mathrm{NaCl} / \mathrm{Cu}(311)$ shows a clear depression at the center of the molecule, indicating a nodal plane, DCV5T/ $\mathrm{NaCl} / \mathrm{Cu}(111)$ does not. Apparently, the energetically lowest-lying state is not the same for the two cases but $\mathrm{S}$ for $\mathrm{DCV} 5 \mathrm{~T} / \mathrm{NaCl} / \mathrm{Cu}(111)$ and $\mathrm{AS}$ in the case of DCV5T/ $\mathrm{NaCl} / \mathrm{Cu}(311)$. In contrast, STM images acquired at voltages well below the first resonance reflect the geometry of the molecule in both cases as wirelike protrusion [see insets of Fig. 1(b)].

We hence assume that the molecules are neutral on $\mathrm{NaCl} / \mathrm{Cu}(111)$ and that the $\mathrm{S}$ state corresponds to the LUMO. According to the literature, changing the copper surface orientation from $\mathrm{Cu}(111)$ to $\mathrm{Cu}(311)$ results in a lowering of the work function by approximately $1 \mathrm{eV}$ [31-33]. Hence, one may expect that the former LUMO, initially located $0.7 \mathrm{eV}$ above the Fermi level $E_{F}$ in the case of $\mathrm{NaCl} / \mathrm{Cu}(111)$, will shift to below the Fermi level $[24,54]$ for $\mathrm{NaCl} / \mathrm{Cu}(311)$ such that the molecule becomes permanently charged.

To obtain a systematic understanding of the level alignment of the $\mathrm{S}$ and AS states of the molecule on both substrates, we acquired differential conductance $(d I / d V)$ spectra and $d I / d V$ maps on DCV5T molecules. Typical spectra measured at the center and the side of the molecule are shown in Figs. 2(a) and 2(b) on $\mathrm{NaCl} / \mathrm{Cu}(111)$ and $\mathrm{NaCl} / \mathrm{Cu}(311)$, respectively. DCV5T exhibits two $d I / d V$ resonances at positive bias but none at negative voltages down to $-2.5 \mathrm{~V}$. According to the $d I / d V$ maps and consistent with the different intensities in the spectra acquired on and off center of the molecule, the $\mathrm{S}$ state at $\simeq 0.7 \mathrm{~V}$ is lower in energy than the AS state occurring at $\simeq 1.1 \mathrm{~V}$. The energy difference of $\simeq 0.4 \mathrm{eV}$ is in rough agreement to our calculations [see Fig. 1(a)]. As discussed above, in the case of $\mathrm{NaCl} / \mathrm{Cu}(311)$, DCV5T exhibits the AS state as the lowest resonance at positive bias voltages, this time at $\simeq 0.9 \mathrm{~V}$. This is additionally evidenced by the constant-current STM image and the corresponding $d I / d V$ 


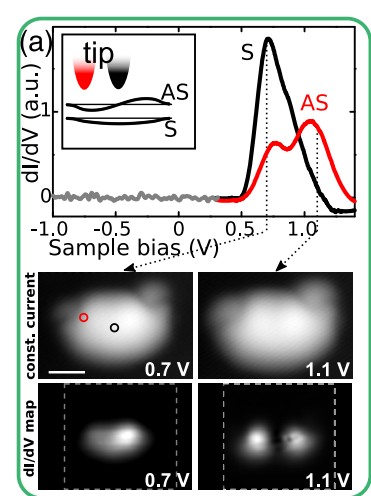

DCV5T/ NaCl/ Cu(111)

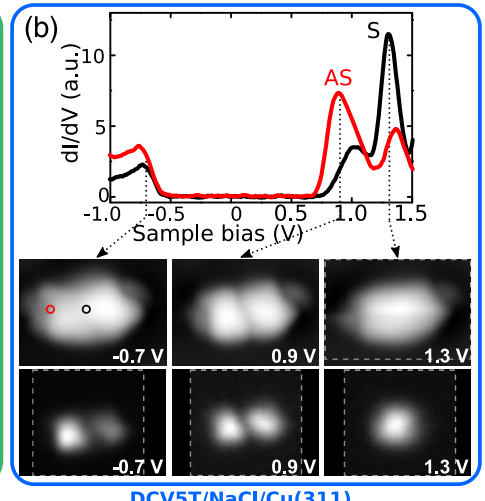

FIG. 2. $d I / d V$ spectra (top panels), constant-current STM images (center panels), and $d I / d V$ maps (bottom panels) on the individual molecule DCV5T on $\mathrm{NaCl} / \mathrm{Cu}(111)$ (a) and $\mathrm{NaCl} / \mathrm{Cu}(311)$ (b) respectively. The resonances are labeled with $\mathrm{S}$ and $\mathrm{AS}$, referring to the symmetic and antisymmetric states, respectively. $d I / d V$ spectra were recorded on (black) and off (red) the center of the molecule as indicated by dots in the STM images. The spatial distribution of orbitals gives rise to the different intensities at different tip positions as depicted in the inset. To not miss any small $d I / d V$ signals in the low-bias range, a corresponding spectrum (gray) was measured with the tip being $\simeq 2 \AA$ closer to the surface compared to the other two (red and black). All spectra were slightly low-pass filtered. The images are resized to have the same size and scale, whereby the area of measured data is indicated with white dashed rectangles. Constant current images $I=2.4 \mathrm{pA}$; bias voltage as indicated. Scale bar $1 \mathrm{~nm}$.

map in Fig. 2(b). The $S$ state is now located at higher voltages, namely at $\simeq 1.3 \mathrm{~V}$, as seen in the spectrum and the $d I / d V$ map. Obviously, the two states are reversed in their sequence. In this case, at negative bias voltages, a peak in $d I / d V$ indicates an occupied state in equilibrium, in stark contrast to $\mathrm{DCV} 5 \mathrm{~T} / \mathrm{NaCl} / \mathrm{Cu}(111)$ but in agreement with the assumption of the molecule being negatively charged. The constant-current image acquired at $-0.7 \mathrm{~V}$, corresponding to the first peak at negative bias, seems to be a superposition of both the S and AS states.

The experimentally observed reversal of the orbital sequence is in striking disagreement with the fundamental oscillation theorem. To understand this apparent orbital reversal, we go beyond the single-particle picture and invoke the role of electronic correlations. In the doublebarrier tunneling junction geometry employed here, the resonances in $d I / d V$ are associated with a temporary change of electron number on the molecule. Therefore, the two peaks of DCV5T/ $\mathrm{NaCl} / \mathrm{Cu}(111)$ at positive bias are DCV5T $\leftrightarrow$ DCV5T $^{-}$transitions (See Fig. 3), and, in the same spirit, the ones of DCV5T/NaCl/Cu(311) at positive and at negative bias should be interpreted as

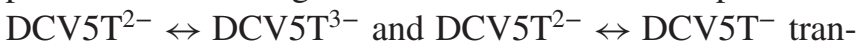
sitions, respectively.

Both the topographical and the spectroscopic data presented so far suggest that the electronic transport

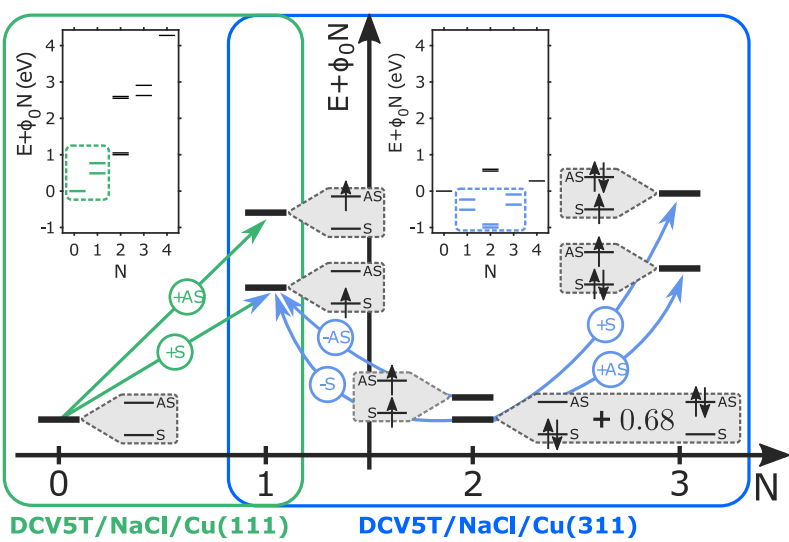

FIG. 3. Scheme with the many-body transitions associated to the measured resonances. The green-framed panel illustrates the transition between the neutral and the singly charged DCV5T ${ }^{-}$ for $\mathrm{DCV} 5 \mathrm{~T} / \mathrm{NaCl} / \mathrm{Cu}(111)$. The blue-framed panel shows the transitions involving DCV5T ${ }^{-}, \mathrm{DCV}^{2-} \mathrm{T}^{2-}$, and $\mathrm{DCV}^{3} \mathrm{~T}^{3-}$ for DCV5T/ $\mathrm{NaCl} / \mathrm{Cu}(311)$. The electronic structure associated to the different many-body states is given in the gray labels. In the insets, the many-body spectra of the molecule on the two corresponding substrates are plotted.

through DCV5T involves, in the present bias and work function ranges, only the symmetric (S) and the antisymmetric (AS) orbitals. We concentrate on them and freeze the occupation of the other lower (higher) energy orbitals to 2 (0). In terms of these S and AS frontier orbitals, we write the minimal interacting Hamiltonian for the isolated molecule

$$
\begin{aligned}
H_{\mathrm{mol}}= & \epsilon_{\mathrm{S}} \hat{n}_{\mathrm{S}}+\epsilon_{\mathrm{AS}} \hat{n}_{\mathrm{AS}}+\frac{U}{2} \hat{N}(\hat{N}-1) \\
& +J \sum_{\sigma \sigma^{\prime}} d_{\mathrm{AS} \sigma}^{\dagger} d_{\mathrm{S} \sigma^{\prime}}^{\dagger} d_{\mathrm{AS} \sigma^{\prime}} d_{\mathrm{S} \sigma} \\
& +J\left(d_{\mathrm{AS} \uparrow}^{\dagger} d_{\mathrm{AS} \downarrow}^{\dagger} d_{\mathrm{S} \downarrow} d_{\mathrm{S} \uparrow}+d_{\mathrm{S} \uparrow}^{\dagger} d_{\mathrm{S} \downarrow}^{\dagger} d_{\mathrm{AS} \downarrow} d_{\mathrm{AS} \uparrow}\right),
\end{aligned}
$$

where $d_{\mathrm{S}(\mathrm{AS}) \sigma}^{\dagger}$ creates an electron with spin $\sigma$ in the symmetric (antisymmetric) orbital, $\hat{n}_{i}$ counts the number of electrons in the orbital with $i=\mathrm{S}, \mathrm{AS}$, and $\hat{N}$ represents the total number of electrons occupying the two frontier orbitals. The interaction parameters $U=1.4 \mathrm{eV}$ and $J=$ $0.75 \mathrm{eV}$ are obtained from the DFT orbitals by direct calculation of the associated Coulomb integrals and assuming a dielectric constant $\epsilon_{r}=2$, which accounts for the screening introduced by the underlying frozen orbitals $[19,56]$. As expected from their similar (de)localization, the Coulomb integrals of the S and AS states are almost identical [57]. Besides a constant interaction charging energy $U$, the model defined in Eq. (1) contains exchange interaction and pair-hopping terms, both proportional to $J$, which are responsible for the electronic correlation. The electrostatic interaction with the substrate is known to stabilize charges on atoms and molecules $[53,58,59]$ due to 
image charge and polaron formation. We account for this stabilization with the additional Hamiltonian $H_{\text {mol-env }}=$ $-\delta \hat{N}^{2}$. The orbital energies $\epsilon_{\mathrm{S}}=-3.1 \mathrm{eV}$ and $\epsilon_{\mathrm{AS}}=$ $-2.8 \mathrm{eV}$ as well as the image-charge renormalization $\delta=0.43 \mathrm{eV}$ are obtained from the experimental resonances of the neutral molecule and previous experimental results on other molecules [29].

Many-body interaction manifests itself most strikingly for the ground state DCV5T ${ }^{2-}$, which will therefore, be discussed at first. Consider the two many-body states, in which the two extra electrons both occupy either the $S$ or the AS state: they differ in energy by the energy $2 \Delta$, where $\Delta=\epsilon_{\mathrm{AS}}-\epsilon_{\mathrm{S}}$ is the single-particle level spacing between the $\mathrm{S}$ and the AS state. These two many-body states interact via pair hopping of strength $J$, leading to a level repulsion. As long as $\Delta \gg J$, this effect is negligible. In DCV5T, though, the single-particle level spacing $\Delta$ is small compared to the pair-hopping $J$, leading to an entangled ground state of DCV5T ${ }^{2-}$ as

$|2,0\rangle=\cos \theta d_{\mathrm{S} \uparrow}^{\dagger} d_{\mathrm{S} \downarrow}^{\dagger}|0,0\rangle+\sin \theta d_{\mathrm{AS} \uparrow}^{\dagger} d_{\mathrm{AS} \downarrow}^{\dagger}|0,0\rangle$,

with $|N, m\rangle$ the $m^{\text {th }}$ excited $N$ particle state of DCV5T, and $\theta=\arctan (J / \Delta) / 2$. Note that here, as $J / \Delta \approx 2.6$, this state shows more than $30 \%$ contribution from both constituent states, is strongly entangled, and therefore, it can not be approximated by a single Slater determinant. The first excited state of DCV5T ${ }^{2-}$ is a triplet with one electron in the $\mathrm{S}$ and one in the AS orbital at about $54 \mathrm{meV}$ above the ground state, as shown in Fig. 3.

The level repulsion in DCV5T ${ }^{2-}$ mentioned above leads to a significant reduction of the ground state energy by roughly $0.5 \mathrm{eV}$. This effect enhances the stability of the doubly charged molecule to the disadvantage of DCV5T-, which has just a single extra electron and therefore, does not feature many-body effects. Quantitatively, this is captured by the addition energy [29].

Within the framework of the many-body theory, as sketched in Fig. 3, the apparent orbital reversal between Fig. 2(a) and Fig. 2(b) is naturally explained. To this end, tunneling events in the STM experiments have to be considered as transitions between the many-body states of different charges $N$ (see arrows in Fig. 3). The spatial fingerprints of the transitions and hence, their appearance in STM images is given by the orbital occupation difference between the two many-body states and is indicated by the labels $\mathrm{S}$ and $\mathrm{AS}$ in Fig. 3. When on $\mathrm{NaCl}(2 \mathrm{ML}) / \mathrm{Cu}(111)$, the DCV5T molecule is in its neutral ground state (Fig. 3, green panel). A sufficiently large positive sample bias triggers transitions to the singly charged DCV5T' and AS transitions become energetically available in the expected order of the corresponding single-particle states. A fast tunneling of the extra electron to the substrate restores the initial condition enabling a steady-state current.

When on $\mathrm{NaCl}(2 \mathrm{ML}) / \mathrm{Cu}(311)$, the molecule is doubly charged and in the entangled ground state described by Eq. (2), see Fig. 3. At sufficiently high positive sample bias, the transitions to DCV5T ${ }^{3-}$ are opening, enabling electron tunneling from the tip to the molecule. The topography of these transitions is again obtained by comparing the twoand the three- (excess) electron states of DCV5T (cf. Fig. 3). The transition to the three-particle ground state occurs by the population of the AS state, and it involves the first component of the entangled two-electron ground state only. The second component cannot contribute to this transition, which is bound to involve only a singleelectron tunneling event. Correspondingly, at a larger bias, the first excited three-particle state becomes accessible, via a transition involving the second component of the twoparticle ground state only. This transition has an S state topography. Hence, although the electronic structure of the three-electron states does follow the Aufbau principle, the entanglement of the two-particle ground state leads to the apparent reversal of the orbital sequence.

As described in the Supplemental Material [29], in addition to the many-body spectrum, we calculated the full dynamics of subsequent tunneling processes for all relevant situations, resulting in the calculated $d I / d V$ characteristics, constant current maps, and constant height $d I / d V$ maps for a DCV5T single-molecule junction presented in Fig. 4. A qualitative agreement with the experimental results of Fig. 2 can be observed both for the relative strength of the spectral peaks and the $d I / d V$ maps. The observed apparent orbital reversal is fully consistent with the calculations.

The experimental data of DCV5T on the $\mathrm{Cu}(311)$ substrate at negative bias also show a nonstandard feature. The $d I / d V$ map at resonance resembles a superposition of the S and AS orbital, see Fig. 2(b). The effect is also

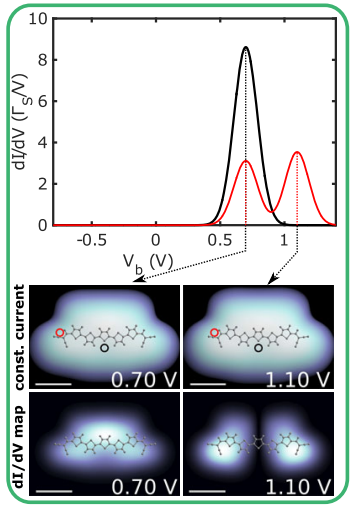

DCV5T/NaCl/Cu(111)

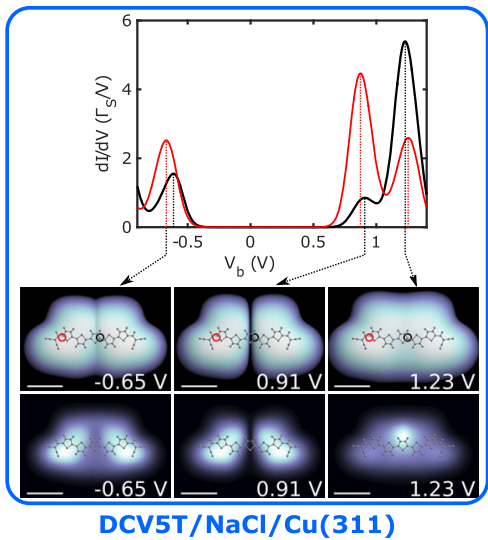

FIG. 4. Theoretical simulations of $d I / d V$ spectra (top panels), constant-current STM images (center panels), and $d I / d V$ maps (bottom panels) on the individual molecule DCV5T on $\mathrm{NaCl} /$ $\mathrm{Cu}(111)$ (left) and $\mathrm{NaCl} / \mathrm{Cu}(311)$ (right), respectively. $d I / d V$ spectra were recorded on (black) and off (red) center of the molecule as indicated by dots in the constant-current STM images. 
reproduced in the theoretical simulations presented in Fig. 4 and is discussed in the Supplemental Material [29] in terms of a nonequilibrium dynamics associated to a population inversion recently predicted [19].

In conclusion, we showed that a reduction of the singleparticle level spacing engineered by dicyanovinyl substitution is leading to an apparent reversal of orbital sequence and a strongly entangled ground state of DCV5T ${ }^{2-}$. The many-body description of the electronic transport is capable to reconcile the experimental observations of the orbital reversal with the fundamental oscillation theorem of quantum mechanics. It shows how to achieve quantum entanglement of frontier orbitals in molecules by chemical design with implications far beyond the particular molecule studied here.

The authors thank Milena Grifoni for valuable comments and discussions and David Kasipović for help. Financial support from the Deutsche Forschungsgemeinschaft via SFB 689 and GRK 1570 and the Volkswagen Foundation through its Lichtenberg program are gratefully acknowledged.

*jascha.repp@ur.de †andrea.donarini@ur.de

[1] D. M. Cardamone, C. A. Stafford, and S. Mazumdar, Nano Lett. 6, 2422 (2006).

[2] A. Donarini, G. Begemann, and M. Grifoni, Nano Lett. 9, 2897 (2009).

[3] A. Donarini, G. Begemann, and M. Grifoni, Phys. Rev. B 82, 125451 (2010).

[4] C. M. Guédon, H. Valkenier, T. Markussen, K. S. Thygesen, J. C. Hummelen, and S. J. van der Molen, Nat. Nanotechnol. 7, 305 (2012).

[5] H. Vazquez, R. Skouta, S. Schneebeli, M. Kamenetska, R. Breslow, L. Venkataraman, and M. Hybertsen, Nat. Nanotechnol. 7, 663 (2012).

[6] S. Ballmann, R. Härtle, P. B. Coto, M. Elbing, M. Mayor, M. R. Bryce, M. Thoss, and H. B. Weber, Phys. Rev. Lett. 109, 056801 (2012).

[7] J. Xia, B. Capozzi, S. Wei, M. Strange, A. Batra, J. R. Moreno, R. J. Amir, E. Amir, G. C. Solomon, L. Venkataraman, and L. M. Campos, Nano Lett. 14, 2941 (2014).

[8] A. Zhao, Q. Li, L. Chen, H. Xiang, W. Wang, S. Pan, B. Wang, X. Xiao, J. Yang, J. G. Hou, and Q. Zhu, Science 309, 1542 (2005).

[9] G. Maruccio, M. Janson, A. Schramm, C. Meyer, T. Matsui, C. Heyn, W. Hansen, R. Wiesendanger, M. Rontani, and E. Molinari, Nano Lett. 7, 2701 (2007).

[10] I. Fernández-Torrente, K. J. Franke, and J. I. Pascual, Phys. Rev. Lett. 101, 217203 (2008).

[11] K. J. Franke, G. Schulze, and J. I. Pascual, Science 332, 940 (2011).

[12] A. Chiesa, S. Carretta, P. Santini, G. Amoretti, and E. Pavarini, Phys. Rev. Lett. 110, 157204 (2013).

[13] S. Grothe, S. Johnston, S. Chi, P. Dosanjh, S. A. Burke, and Y. Pennec, Phys. Rev. Lett. 111, 246804 (2013).
[14] M. M. Ervasti, F. Schulz, P. Liljeroth, and A. Harju, J. Electron Spectrosc. Relat. Phenom, DOI: 10.1016/j. elspec.2016.11.004 (2016).

[15] G. Begemann, D. Darau, A. Donarini, and M. Grifoni, Phys. Rev. B 77, 201406(R) (2008).

[16] D. Toroz, M. Rontani, and S. Corni, J. Chem. Phys. 134, 024104 (2011).

[17] D. Toroz, M. Rontani, and S. Corni, Phys. Rev. Lett. 110, 018305 (2013).

[18] F. Schulz, M. Ijäs, R. Drost, S. K. Hämäläinen, A. Harju, A. P. Seitsonen, and P. Liljeroth, Nat. Phys. 11, 229 (2015).

[19] B. Siegert, A. Donarini, and M. Grifoni, Phys. Rev. B 93, 121406(R) (2016).

[20] M.-S. Liao and S. Scheiner, J. Chem. Phys. 114, 9780 (2001).

[21] K. A. Nguyen and R. Pachter, J. Chem. Phys. 118, 5802 (2003).

[22] S. W. Wu, N. Ogawa, and W. Ho, Science 312, 1362 (2006).

[23] S. W. Wu, N. Ogawa, G. V. Nazin, and W. Ho, J. Phys. Chem. C 112, 5241 (2008).

[24] C. Uhlmann, I. Swart, and J. Repp, Nano Lett. 13, 777 (2013).

[25] E. Hückel, Z. Phys. 70, 204 (1931).

[26] W. Barford, Electronic and Optical Properties of Conjugated Polymers (Oxford University Press, Oxford, 2005).

[27] F. J. Giessibl, Appl. Phys. Lett. 76, 1470 (2000).

[28] J. M. Soler, E. Artacho, J. D. Gale, A. García, J. Junquera, P. Ordejón, and D. Sánchez-Portal, J. Phys. Condens. Matter 14, 2745 (2002).

[29] See Supplemental Material at http://link.aps.org/ supplemental/10.1103/PhysRevLett.119.056801, which includes Refs. [30-48], for more experimental details and further information on the setup of the generalized master equation and on the parametrization of $H_{\text {mol }}$ and $H_{\text {mol-env }}$.

[30] R. Fitzner, E. Reinold, A. Mishra, E. Mena-Osteritz, H. Ziehlke, C. Körner, K. Leo, M. Riede, M. Weil, O. Tsaryova, A. Weiss, C. Uhrich, M. Pfeiffer, and P. Bäuerle, Adv. Funct. Mater. 21, 897 (2011).

[31] J. Repp, G. Meyer, S. M. Stojković, A. Gourdon, and C. Joachim, Phys. Rev. Lett. 94, 026803 (2005).

[32] P. O. Gartland, S. Berge, and B. Slagsvold, Phys. Rev. Lett. 28, 738 (1972).

[33] F. E. Olsson, M. Persson, J. Repp, and G. Meyer, Phys. Rev. B 71, 075419 (2005).

[34] S. Sobczyk, A. Donarini, and M. Grifoni, Phys. Rev. B 85, 205408 (2012).

[35] A. Donarini, B. Siegert, S. Sobczyk, and M. Grifoni, Phys. Rev. B 86, 155451 (2012).

[36] B. Siegert, A. Donarini, and M. Grifoni, Phys. Status Solidi (b) 250, 2444 (2013).

[37] D. Darau, G. Begemann, A. Donarini, and M. Grifoni, Phys. Rev. B 79, 235404 (2009).

[38] J. Koch and F. von Oppen, Phys. Rev. Lett. 94, 206804 (2005).

[39] R. Bennewitz, M. Bammerlin, M. Guggisberg, C. Loppacher, A. Baratoff, E. Meyer, and H.-J. Güntherodt, Surf. Interface Anal. 27, 462 (1999).

[40] M. Nonnenmacher, Appl. Phys. Lett. 58, 2921 (1991).

[41] S. Sadewasser, P. Jelínek, C.-K. Fang, O. Custance, Y. Yamada, Y. Sugimoto, M. Abe, and S. Morita, Phys. Rev. Lett. 103, 266103 (2009). 
[42] F. Mohn, L. Gross, N. Moll, and G. Meyer, Nat. Nanotechnol. 7, 227 (2012).

[43] W. Steurer, J. Repp, L. Gross, I. Scivetti, M. Persson, and G. Meyer, Phys. Rev. Lett. 114, 036801 (2015).

[44] L. Gross, F. Mohn, P. Liljeroth, J. Repp, F. J. Giessibl, and G. Meyer, Science 324, 1428 (2009).

[45] B. Schuler, S.-X. Liu, Y. Geng, S. Decurtins, G. Meyer, and L. Gross, Nano Lett. 14, 3342 (2014).

[46] J. L. Neff and P. Rahe, Phys. Rev. B 91, 085424 (2015).

[47] F. Albrecht, J. Repp, M. Fleischmann, M. Scheer, M. Ondráček, and P. Jelínek, Phys. Rev. Lett. 115, 076101 (2015).

[48] M. Ikeda, N. Koide, L. Han, A. Sasahara, and H. Onishi, J. Phys. Chem. C 112, 6961 (2008).

[49] W. J. Feast, J. Tsibouklis, K. L. Pouwer, L. Groenendaal, and E. W. Meijer, Polymer 37, 5017 (1996).

[50] R. Yamada, H. Kumazawa, T. Noutoshi, S. Tanaka, and H. Tada, Nano Lett. 8, 1237 (2008).
[51] J. Repp, P. Liljeroth, and G. Meyer, Nat. Phys. 6, 975 (2010).

[52] D. A. Kislitsyn, B. N. Taber, C. F. Gervasi, L. Zhang, S. C. B. Mannsfeld, J. S. Prell, A. Briseno, and G. V. Nazin, Phys. Chem. Chem. Phys. 18, 4842 (2016).

[53] F. E. Olsson, S. Paavilainen, M. Persson, J. Repp, and G. Meyer, Phys. Rev. Lett. 98, 176803 (2007).

[54] I. Swart, T. Sonnleitner, and J. Repp, Nano Lett. 11, 1580 (2011).

[55] L. Bogner, Z. Yang, M. Corso, R. Fitzner, P. Bäuerle, K. J. Franke, J. I. Pascual, and P. Tegeder, Phys. Chem. Chem. Phys. 17, 27118 (2015).

[56] D. A. Ryndyk, A. Donarini, M. Grifoni, and K. Richter, Phys. Rev. B 88, 085404 (2013).

[57] For the Coulomb integrals, we obtain $U_{\mathrm{S}-\mathrm{S}}=1.37 \mathrm{eV}$, $U_{\mathrm{AS}-\mathrm{AS}}=1.43 \mathrm{eV}, U_{\mathrm{S}-\mathrm{AS}}=1.37 \mathrm{eV}$.

[58] K. Kaasbjerg and K. Flensberg, Nano Lett. 8, 3809 (2008).

[59] K. Kaasbjerg and K. Flensberg, Phys. Rev. B 84, 115457 (2011). 